\title{
ON SHARP PERTURBED MIDPOINT INEQUALITIES
}

\author{
ZHENG LIU
}

\begin{abstract}
The main purpose of this paper is to use a variant of Grüss inequality to obtain some perturbed midpoint inequalities. Moreover, we show that our results are sharp and precisely characterize the functions for which equality holds. Thus we provide improvements of some recent results for perturbed midpoint inequalities.
\end{abstract}

\section{Introduction}

In 1935, G. Grüss proved the following integral inequality which gives an approximation for the integral of the product of two functions in terms of the product of the integrals of the two functions (see for example [6, p.296]).

Theorem A. Let $h, g:[a, b] \rightarrow \boldsymbol{R}$ be two integrable functions such that $\phi \leq h(x) \leq \Phi$ and $\gamma \leq g(x) \leq \Gamma$ for all $x \in[a, b]$, where $\phi, \Phi, \gamma$ and $\Gamma$ are real numbers. Then we have

$$
\begin{aligned}
|T(h, g)| & :=\left|\frac{1}{b-a} \int_{a}^{b} h(x) g(x) d x-\frac{1}{b-a} \int_{a}^{b} h(x) d x \cdot \frac{1}{b-a} \int_{a}^{b} g(x) d x\right| \\
& \leq \frac{1}{4}(\Phi-\phi)(\Gamma-\gamma),
\end{aligned}
$$

and the inequality is sharp, in the sense that the constant $\frac{1}{4}$ can not be replaced by a smaller one.

It is clear that the constant $\frac{1}{4}$ is achieved for

$$
h(x)=g(x)=\operatorname{sgn}\left(x-\frac{a+b}{2}\right) .
$$

From then on, (1) is well known in the literature as Grüss inequality.

In 2000, M. Matić, J. Pečarić and N. Ujević [5] established the following inequality of Grüss type:

Received October 17, 2003.

2000 Mathematics Subject Classification. 26D15.

Key words and phrases. Grüss inequality, midpoint inequality, perturbed midpoint inequality. 
Theorem B. Let $h, g:[a, b] \rightarrow \boldsymbol{R}$ be integrable functions such that $h g$ is also integrable, and $\gamma \leq g(x) \leq \Gamma$ for all $x \in[a, b]$, where $\gamma, \Gamma \in \boldsymbol{R}$ are constants. Then

$$
|T(h, g)| \leq \frac{1}{2} \sqrt{T(h, h)}(\Gamma-\gamma)
$$

(2) is also called as a premature Grüss inequality in [1].

In the recent paper [3], X. L. Cheng and J. Sun proved the following variant of the Grüss inequality.

Theorem C. Let $h, g:[a, b] \rightarrow \boldsymbol{R}$ be two inegrable functions such that $\gamma \leq g(x) \leq \Gamma$ for some constants $\gamma, \Gamma$ for all $x \in[a, b]$. Then

$$
\begin{aligned}
& \left|\int_{a}^{b} h(x) g(x) d x-\frac{1}{b-a} \int_{a}^{b} h(x) d x \int_{a}^{b} g(x) d x\right| \\
\leq & \frac{1}{2}\left(\int_{a}^{b}\left|h(x)-\frac{1}{b-a} \int_{a}^{b} h(y) d y\right| d x\right)(\Gamma-\gamma) .
\end{aligned}
$$

Here we have given revised version for (3) since the expression in [3] contained a misprint.

It is not difficult to find that Theorem B improves Theorem A and Theorem $\mathrm{C}$ improves Theorem B.

The following inequality is well known in the literature as the midpoint inequality:

$$
\left|\int_{a}^{b} f(x) d x-(b-a) f\left(\frac{a+b}{2}\right)\right| \leq \frac{(b-a)^{3}}{24}\left\|f^{\prime \prime}\right\|_{\infty}
$$

where the mapping $f:[a, b] \rightarrow \boldsymbol{R}$ is assumed to be twice differentiable on the interval $(a, b)$ and having the second derivative bounded on $(a, b)$. That is, $\left\|f^{\prime \prime}\right\|_{\infty}:=$ $\sup _{x \in(a, b)}\left|f^{\prime \prime}(x)\right| \leq \infty$.

From [2] and [4], we see that by applying the Grüss inequality (1) a perturbed midpoint inequality can be established as follows:

Theorem D. Let $f:[a, b] \rightarrow \boldsymbol{R}$ be such that $f^{\prime \prime}$ is integrable with $\gamma \leq f^{\prime \prime}(x) \leq \Gamma$ for all $x \in[a, b]$, where $\gamma, \Gamma \in \boldsymbol{R}$ are constants. Then we have the perturbed midpoint inequality:

$$
\left|\int_{a}^{b} f(x) d x-(b-a) f\left(\frac{a+b}{2}\right)-\frac{(b-a)^{2}}{24}\left[f^{\prime}(b)-f^{\prime}(a)\right]\right| \leq \frac{\Gamma-\gamma}{4}\left(\frac{b-a}{2}\right)^{3} .
$$

From [1] and [5], we see that by applying the premature Grüss inequality (2) gives a genreal version of perturbed midpoint inequality as follows: 
Theorem E. Let $f:[a, b] \rightarrow \boldsymbol{R}$ be such that $f^{(n)}$ is integrable with $\gamma \leq f^{(n)}(x) \leq \Gamma$ for all $x \in[a, b]$, where $\gamma, \Gamma \in \boldsymbol{R}$ are constants. Then

$$
\begin{aligned}
& \mid \int_{a}^{b} f(x) d x-(b-a) f\left(\frac{a+b}{2}\right)-\sum_{k=1}^{n-1} \frac{1+(-1)^{k}}{(k+1) !}\left(\frac{b-a}{2}\right)^{k+1} f^{(k)}\left(\frac{a+b}{2}\right) \\
& \quad-\frac{1+(-1)^{n}}{(n+1) !}\left(\frac{b-a}{2}\right)^{n+1} \frac{f^{(n-1)}(b)-f^{(n-1)}(a)}{b-a} \mid \\
& \leq \\
& \leq \frac{\Gamma-\gamma}{n ! \sqrt{2 n+1}}\left[1-\frac{1+(-1)^{n}}{2(n+1)}\right]\left(\frac{b-a}{2}\right)^{n+1} .
\end{aligned}
$$

Corollary. Let $f:[a, b] \rightarrow \boldsymbol{R}$ be such that $f^{\prime \prime}$ is integrable with $\gamma \leq f^{\prime \prime}(x) \leq \Gamma$ for all $x \in[a, b]$, where $\gamma, \Gamma \in \boldsymbol{R}$ are constants. Then

$$
\left|\int_{a}^{b} f(x) d x-(b-a) f\left(\frac{a+b}{2}\right)-\frac{(b-a)^{2}}{24}\left[f^{\prime}(b)-f^{\prime}(a)\right]\right| \leq \frac{\Gamma-\gamma}{3 \sqrt{5}}\left(\frac{b-a}{2}\right)^{3} .
$$

Clearly, the perturbed midpoint inequality (7) provides an improvement of the perturbed midpoint inequality (5).

The purpose of this short paper is to provide some sharp perturbed midpoint inequalities by using the variant of the Grüss inequality (3).

\section{Main Result}

To obtain our main result, we need the following integral identity whose proof can be found in $[1]$.

Lemma. Let $f:[a, b] \rightarrow \boldsymbol{R}$ be such that $f^{(n)}$ is integrable. Then we have the identity:

$$
\begin{aligned}
(-1)^{n} \int_{a}^{b} M_{n}(x) f^{(n)}(x) d x= & \int_{a}^{b} f(x) d x-(b-a) f\left(\frac{a+b}{2}\right) \\
& -\sum_{k=1}^{n-1} \frac{1+(-1)^{k}}{(k+1) !}\left(\frac{b-a}{2}\right)^{k+1} f^{(k)}\left(\frac{a+b}{2}\right) .
\end{aligned}
$$

where

$$
M_{n}(x):= \begin{cases}\frac{(x-a)^{n}}{n !}, & \text { if } x \in\left[a, \frac{a+b}{2}\right] \\ \frac{(x-b)^{n}}{n !}, & \text { if } x \in\left(\frac{a+b}{2}, b\right]\end{cases}
$$

Theorem. Let $f:[a, b] \rightarrow \boldsymbol{R}$ be such that $f^{(n)}$ is integrable with $\gamma \leq f^{(n)}(x) \leq \Gamma$ for all $x \in[a, b]$, where $\gamma, \Gamma \in \boldsymbol{R}$ are constants. Then

$$
\mid \int_{a}^{b} f(x) d x-(b-a) f\left(\frac{a+b}{2}\right)-\sum_{k=1}^{n-1} \frac{1+(-1)^{k}}{(k+1) !}\left(\frac{b-a}{2}\right)^{k+1} f^{(k)}\left(\frac{a+b}{2}\right)
$$




$$
\begin{aligned}
& -\frac{1+(-1)^{n}}{(n+1) !}\left(\frac{b-a}{2}\right)^{n+1} \frac{f^{(n-1)}(b)-f^{(n-1)}(a)}{b-a} \mid \\
\leq & \frac{\Gamma-\gamma}{(n+1) !}\left[\frac{1-(-1)^{n}}{2}+\frac{\left(1+(-1)^{n}\right) n}{(n+1) \sqrt[n]{n+1}}\right]\left(\frac{b-a}{2}\right)^{n+1} .
\end{aligned}
$$

Proof. It is clear that

$$
\int_{a}^{b} M_{n}(x) d x=\frac{1+(-1)^{n}}{(n+1) !}\left(\frac{b-a}{2}\right)^{n+1}
$$

Then by (8), (10) and (3) we can derive

$$
\begin{aligned}
& \mid \int_{a}^{b} f(x) d x-(b-a) f\left(\frac{a+b}{2}\right)-\sum_{k=1}^{n-1} \frac{1+(-1)^{k}}{(k+1) !}\left(\frac{b-a}{2}\right)^{k+1} f^{(k)}\left(\frac{a+b}{2}\right) \\
& \quad-\frac{1+(-1)^{n}}{(n+1) !}\left(\frac{b-a}{2}\right)^{n+1} \frac{f^{(n-1)}(b)-f^{(n-1)}(a)}{b-a} \mid \\
& \leq \frac{\Gamma-\gamma}{2} \int_{a}^{b}\left|M_{n}(x)-\frac{1+(-1)^{n}}{2(n+1) !}\left(\frac{b-a}{2}\right)^{n}\right| d x .
\end{aligned}
$$

For an odd $n$, the integral in the right-hand side of inequality (11) is equal to

$$
\int_{a}^{b}\left|M_{n}(x)\right| d x=\frac{2}{(n+1) !}\left(\frac{b-a}{2}\right)^{n+1} .
$$

For an even $n$, the integral in the right-hand side of inequality (11) can be calculated as follows:

Let $x_{1}=a+\frac{1}{\sqrt[n]{n+1}}\left(\frac{b-a}{2}\right)$ and $x_{2}=b-\frac{1}{\sqrt[n]{n+1}}\left(\frac{b-a}{2}\right)$. Then $a<x_{1}<x_{2}<b$ and $x_{1}-a=b-x_{2}=\frac{1}{\sqrt[n]{n+1}}\left(\frac{b-a}{2}\right)$. Thus we have

$$
\begin{aligned}
& \int_{a}^{b}\left|M_{n}(x)-\frac{1}{(n+1) !}\left(\frac{b-a}{2}\right)^{n}\right| d x \\
= & \int_{a}^{\frac{a+b}{2}}\left|\frac{(x-a)^{n}}{n !}-\frac{1}{(n+1) !}\left(\frac{b-a}{2}\right)^{n}\right| d x+\int_{\frac{a+b}{2}}^{b}\left|\frac{(x-b)^{n}}{n !}-\frac{1}{(n+1) !}\left(\frac{b-a}{2}\right)^{n}\right| d x \\
= & \int_{a}^{x_{1}}\left[\frac{1}{(n+1) !}\left(\frac{b-a}{2}\right)^{n}-\frac{(x-a)^{n}}{n !}\right] d x+\int_{x_{1}}^{\frac{a+b}{2}}\left[\frac{(x-a)^{n}}{n !}-\frac{1}{(n+1) !}\left(\frac{b-a}{2}\right)^{n}\right] d x \\
& +\int_{\frac{a+b}{2}}^{x_{2}}\left[\frac{(x-b)^{n}}{n !}-\frac{1}{(n+1) !}\left(\frac{b-a}{2}\right)^{n}\right] d x+\int_{x_{2}}^{b}\left[\frac{1}{(n+1) !}\left(\frac{b-a}{2}\right)^{n}-\frac{(x-b)^{n}}{n !}\right] d x \\
= & \frac{(n-a}{(n+1) !(n+1) \sqrt[n]{n+1}}\left(\frac{b-1}{2}\right)^{n+1} \cdot
\end{aligned}
$$


Consequently, the inequality (9) follows from (11), (12), and (13).

Remark 1. It is not difficult to find that the inequality (9) provides an improvement of the inequality (6). Actually we can prove that the inequality (9) is sharp. Indeed, we can construct the function $f(x)=\int_{a}^{x}\left(\int_{a}^{y_{n}}\left(\cdots \int_{a}^{y_{2}} j\left(y_{1}\right) d y_{1} \cdots\right) d y_{n-1}\right) d y_{n}$ to attain the equality in (9), where

$$
j(x)= \begin{cases}\Gamma, & a \leq x<\frac{a+b}{2}, \\ \gamma, & \frac{a+b}{2} \leq x \leq b,\end{cases}
$$

when $n$ is odd, and

$$
j(x)= \begin{cases}\gamma, & a \leq x<x_{1}=a+\frac{1}{\sqrt[n]{n+1}}\left(\frac{b-a}{2}\right), \\ \Gamma, & x_{1} \leq x<x_{2}=b-\frac{1}{\sqrt[n]{n+1}}\left(\frac{b-a}{2}\right), \\ \gamma, & x_{2} \leq x \leq b\end{cases}
$$

when $n$ is even.

Remark 2. For $n=1$ then there is no perturbation term, giving

$$
\left|\int_{a}^{b} f(x) d x-(b-a) f\left(\frac{a+b}{2}\right)\right| \leq \frac{\Gamma-\gamma}{2}\left(\frac{b-a}{2}\right)^{2},
$$

where $\gamma \leq f^{\prime}(x) \leq \Gamma$. This result may be compared with (4).

If $n=2$ is taken, then there is a perturbation term giving

$$
\left|\int_{a}^{b} f(x) d x-(b-a) f\left(\frac{a+b}{2}\right)-\frac{(b-a)^{2}}{24}\left[f^{\prime}(b)-f^{\prime}(a)\right]\right| \leq \frac{2(\Gamma-\gamma)}{9 \sqrt{3}}\left(\frac{b-a}{2}\right)^{3},
$$

where $\gamma \leq f^{\prime \prime}(x) \leq \Gamma$. Clearly, the perturbed midpoint inequality (14) provides a further improvement of the perturbed midpoint inequality (7). In fact, the constant $\frac{2}{9 \sqrt{3}}$ is sharp in the sense that it cannot be replaced by a smaller one.

\section{References}

[1] P. Cerone and S. S. Dragomir, Midpoint type rules from an inequalities point of view, RGMIA Research Report Collection, 2(1999), No. 7, Article 1.

(http://rgmia.vu.edu.au/v2n7.html).

[2] P. Cerone, S. S. Dragomir and J. Roumeliotis, An inequality of Ostrowski-Grüss type for twice differentiable mappings and applications in numerical integration, Kyungpook Mathematical Journal, 39(1999), 331-341.

[3] X. L. Cheng and J. Sun, A note on the perturbed trapezoid inequality, Journal of Inequalities in Pure and Applied Mathematics, 3(2002), issue 2, Article 29. (http://jipam.vu.edu.au/).

[4] S. S. Dragomir, P. Cerone and A. Sofo, Some remarks on the midpoint rule in numerical integration, Studia Universitatis Babes-Bolyai Mathematica, XLV(1)(2000), 63-74. 
[5] M. Matić, J. Pečarić and N. Ujević, Improvement and further generalization of inequalities of Ostrowski-Grüss type, Computer Math. Appl., 39(2000), 161-175.

[6] D. S. Mitrinović, J. Pečarić and A. M. Fink, Classical and New Inequalities in Analysis, Kluwer Academic Publishers, 1993.

Institute of Applied Mathematics, Faculty of Science, Anshan University of Science and Technology, Anshan 114044, Liaoning, China. 